\title{
The gut in the beaker: missing the surfactants?
}

Clive G Wilson ${ }^{1 *}$, Gavin W. Halbert ${ }^{1}$, Jenifer Mains ${ }^{2}$.

1. Strathclyde Institute of Pharmacy and Biomedical Sciences, University of Strathclyde, Glasgow G4 ORE, Scotland, U.K.

2. EnCap drug delivery, Livingston EH53 OTH, Scotland, U.K.

In this review, we attempt to distill our thoughts regarding the study of the interaction of luminal fluids with the drug-matrix interface, a subject close to Sandy's long-term research interests enveloping excipients, lipids, drug absorption mechanisms and innovative formulation approaches. The interests of our panel of writers, stimulated by some of Professor Florence's earlier work, has prompted us to review how much of the absorption process can be understood in terms of simple physicochemical mechanisms, processes that will allow us to examine the idiosyncrasies of compounds and formulations in complex matrices, representing food and the changing states of secretions. This paper is our contribution to the special edition of the International Journal of Pharmaceutics in honour of Professor Alexander Florence, a friend and a mentor for many years.

The rate of appearance and, in some cases, the total quantity of an active pharmaceutical ingredient found in the systemic blood after oral administration is related to the ability to form a solution of the drug in the contents of the gastrointestinal lumen (Nelson, 1957). Although the physicochemical properties and basic aqueous solubility of the drug are key drivers in this process, it has been appreciated for some time that simple in vitro aqueous solubility is a poor predictor of in vivo gut solubility (Dressman et al., 2007). The biopharmaceutical importance of this discrepancy increases inversely in relation to the dose/solubility ratio of the API, as expressed in the Developability Classification System (Butler \& Dressman 2010). Achieving solubility in poorly mixed gut luminal fluids is a complex phenomenon but two features are important: the drug's physicochemical properties and the influence of both natural and excipient based surfactants in sustaining solubility of the API as the luminal composition changes. Professor Florence has contributed to the study of these aspects and ongoing research has attempted to fill in some of the gaps in our knowledge by the development of new instrumentation. The results from investigations collected with the new technology are being gradually being integrated into in silico models of the dissolution process. The consolidation of 'rules' from the study of dissolution of API in 
luminal fluids is complicated because fluids vary in composition between individuals, influenced by diet, drug exposure and disease. The impact in some cases will be minor whereas for some chemical classes they will be significant. Moreover, the fluids are complex media that alter as they travel down the gut and different structures form. This is especially true for the fed state where additionally, mechanical forces are applied to the food matrix to aid dispersion and increase surface area for enzymatic action. Oral formulations are subjected to altered (increased) mechanical forces when taken with food and therefore such conditions need to be simulated in laboratory-based tests.

The gastrointestinal tract is a muscular tube whose role is the reduction of the food matrix to a slurry, the secretion of enzymes and the absorption of the separated, useful components from chyme. For many nutrients, the absorption is an active process since the products of digestion are small and polar and the nature of the epithelium is a lipoid barrier. Thus absorption is always likely to be slow process, requiring energy and the diversion of blood to transport the nutrients to the liver and beyond. The calorific gain from intake of carbohydrate to fat varies over a three-fold range. The components embedded in meat, grain and fat from this omnivorous diet taken in as a single meal is stored in the stomach to be supplied to the intestine at an optimised rate. Through hormonal mechanisms and sampling high in the gastrointestinal tract, the contact time with the absorbing membrane is regulated to facilitate maximum efficiency of digestion according to the size and type of meal. In addition, the components may require extensive mastication or mixing movements of muscle to lubricate the food mass and to allow more dispersion. Not too far back in our evolution, man was a nomadic hunter-gatherer and warrior, and food was distinctly unprocessed and fibre rich. The gut therefore had to work hard to separate useful material from a diverse matrix and protect against the occasional poisons from the seeds of berries and fruits which might be accidentally ingested. The evolution of the gastrointestinal tract has resulted into a gut specialized into segments, with an acid sump to coagulate proteins followed by a duodenum in which the luminal contents are adjusted towards neutrality and bile added to emulsify fats. The dispersed material is then digested, resulting in a gradual loss of volume leaving some useful but 'locked' nutrients to be extracted through bacterial action. To shield the liver from insult, behavioral and sensory mechanisms developed to avoid retention of poisons; 
transporter mechanisms became evident to reduce exposure and detoxifying enzymes inducible to aid excretion of the xenobiotics.

It can be immediately appreciated that the gut is actively monitoring the composition of ingested material. Drugs have almost no nutritional value and mechanisms that help us to protect ourselves from poisons come into play: specifically taste and the vomiting reflex have to be intentionally bypassed by disguise of the API in a formulation. That work then has to be undone further down the gut to achieve the anticipated drug concentration -time exposure.

The analytical task we face is how to make continuous measurements in a murky media, whose composition changes along the gastrointestinal tract (Dressman et al., 2007). Within the darkness of the gut, phenomena such as disintegration, supersaturation, precipitation and dissolution are occurring in unidentified segments. The impact of variability is to necessitate the performance of expensive clinical trials and to slow medicine development, a situation that should be avoided if necessary. The simulated biological fluids that are used in pharmaceutical sciences are evolving recipes and for some media, considerable differences exist as exemplified by the formulae, as reviewed by Marques and colleagues. For example, simulated saliva, which in five recipes, differs in electrolyte composition and the presence or absence of mucin (Marques et al., 2011). The importance of mucin is more apparent in considering a food matrix rather than medications, since salivary mucin induces flocculation of some emulsions, probably changing sensory perception (Vingerhoeds et al., 2005). It is however essential to assist the normal swallowing reflex.

\section{Tasting and judging whether you can swallow a medicine.}

Except for specially designed formulations, which may be administered to bypass the first pass effect or to directly treat the mouth, an early task is to assess the acceptability of the dosage form. We apply different judgments according to the physical state of the material. For example, a strange colored clear liquid will be sampled by judging acidity, saltiness, bitterness, sweetness, effervescence, the presence of off-flavors and the smell that we perceive during ingestion. The process of swallowing involves a voluntary oral phase, where the tongue samples the ingested material and the person makes a decision about whether it can be swallowed. Difficulty in swallowing 
medications is a commonly reported problem but although phagophobia, a perceived risk of choking in the absence of a physiological explanation is rare in patients, it has been reported in HIV sufferers who have a high oral dose burden (Duggan et al., 2014). Saliva needs to be present as a lubricant and pools in the mouth below the tongue and on the cheek- gum boundary. The dosage form is usually moistened by the action of the tongue pressing the dosage form against the palate and then moving it backwards, contained in a depression formed in the tongue. Food is usually chewed and moistened with saliva to form a bolus for swallowing and if the tongue senses the food is too dry; the swallow will be aborted. The wetted bolus is presented to the back of the mouth, stimulating the palatal receptors and touch receptors of the pharynx (Gleeson, 1999). Pharyngeal peristalsis imparts a forward velocity of a swallowed bolus $>0.3 \mathrm{~m} / \mathrm{s}$ as measured by video fluoroscopy. In patients with dysphagia, increasing the viscosity of the bolus to 'pudding consistency' ( $3682 \mathrm{mPa}$.s) was associated with more efficient swallowing (Clavé et al., 2008). Surprisingly, thin liquids present special problems for patients with neurological disease. For solid formulations that will be swallowed, still more decisions based on shape factors and surface feel become at issue.

As yet, our success in replicating mouth sensations is limited. Taste perception is modulated by various factors including aging, gender, ethnicity, cigarette smoking, olfactory stimuli [as well as the temporary loss of smell sense during a cold], time of day and even psychological processes. As we get older, we lose the ability to detect salty and bitter substances whereas the thresholds for sour or sweet stimuli appear to be unchanged up to the age of 90 (Mojet et al., 2001).

\section{Swallowing medicines}

At the present time, we do not have in vitro systems for testing the ability to swallow a formulation. Professor Florence was interested in the phenomenon of tablet adhesion, especially precipitated by concerns relating to early osmotic tablet formulations. Swisher and colleagues at ALZA described a method of evaluating adhesion of tablets and capsules to the isolated dog and pig oesophagus and ranked the forces of adherence for different formulations (Swisher et al., 1984). Florence and colleagues used the same methodology at 
Strathclyde University to examine tissue adhesion of tablets and hard-shell capsules showing that film coated tablets had low adhesion (Al-Dujaili, 1986). These data are generally supported by in-vivo measurements in man using gamma scintigraphy. As we age, there is a noticeable decrease in the efficiency of muscular coordination, coupled with muscle weakness tendon stiffness and a loss of motor control. Significantly, impaired transit in the elderly can be readily demonstrated scintigraphically which is of particular relevance for formulations containing irritant drugs (Perkins et al. 1994; ibid 2001). Dr. Stegeman has made an extensive study of the problems in this age group with regard to swallowing medicines and has proposed that attention to appropriate dosage forms for the elderly will have to be provided in the future (Stegeman, 2010).

\section{The stomach}

Functionally the stomach divides into two chambers on eating, the upper part (the fundus) relaxing to accommodate the food. In the body, propagating muscular contractions becoming stronger below the midline of the stomach cause contractions against a partially closed pyloric sphincter, sieving solids and liquids. Acid is formed by secretion aided by the increased hormonally driven blood flow. The duodenum plays a role as a sensor and by modulating the wall pressure difference between the duodenum and the pylorus, the dispersed material enters as an approximately isocaloric load.

After a meal, the matrix distributed in the stomach is non-uniform, with channels of liquid travelling around consolidating food masses, which are stacked from the greater curvature and as food is digested, small particles empty with the liquid phase. It has been generally accepted that preparations that dissolve or form particles of less than 2 $\mathrm{mm}$ in diameter pass through the stomach in a similar fashion to liquids and are less likely to be influenced by gastroparesis (Pohle \& Domschke 2003). Particles can empty as boluses, mix in with the food, or partially stratify according to when during the meal the formulations are consumed (O'Reilly et al., 1987).

All fluids in the gut have a lower surface tension than water and Fell \& Mohammed reported that gastric juice was more effective than bile at wetting a powder sample of phenobarbitone (Fell \& Mohammed, 1995), an extension of the observations almost a decade earlier of 
Finholt \& Solvang, who described the role of surface tension on dissolution kinetics of phenacetin and phenobarbitone in polysorbate-hydrochloric acid media (Finholt \& Solvang, 1968). At the same time as John Fell's work in Manchester, Efentakis and Dressman were rediscovering some of the data known to gastroenterologists and applying it to biopharmaceutical purposes. They reported that aspirated gastric samples had a $\mathrm{pH}$ between 1 and 2 with a measured surface tension of between 35 and $45 \mathrm{mM} / \mathrm{m}$ (Efentakis and Dressman, 1998).

For a drug formulation, the key factors are a dynamic change in the media reflecting the balance of dilution, secretions and buffering by the food (especially protein) and the erosive forces which are applied during trituration of intact objects by contractions in the distal stomach. These conditions are not mimicked in any compendial apparatus and in order to get a more realistic simulation of the conditions in the human stomach in a physical simulator, there must be attention to the nature of the gut fluids, the volume changes, the $\mathrm{pH}$ gradient occurring in the stomach and the periodic forces applied to the formulation. During emptying, the stomach changes shape although this factor is rarely considered; it is however of importance for floating dosage forms.

There have been many attempts to mimic the upper gastrointestinal tract. First this was because it was seen as the easiest task: the stomach can be directly observed during endoscopy and we can sample the stomach and the duodenal contents through a tube. Measurements of fluids further down the Gl tract require more invasive clinical attention. The residence time in the stomach is an important determination determinant of the onset of the arrival of the drug in the plasma and therefore drug action. In addition, disintegration in the stomach might result in dose dumping (especially in the presence of food) and the degradation of drug in gastric acid prior to absorption. Simple models are based on a fixed volume compartment; for example, the modified Rossett and Rice apparatus used by our group to test floating antacids (Washington et al., 1985). More complex models join multiple compartments, arranging transfer of fluid across conditions representing gastro-duodenal exchange with changes in $\mathrm{pH}$. Others apply force periodically to the formulation for example the TNO simulator (TIM-1). One apparatus described by Garbacz and colleagues applies periodic pressure and takes the formulation in and out of contact with fluid (Garbacz et al., 2008). In most of these systems, complex media can be used but there is always an issue 
with regard to turbidity and media which have high optical absorbance. In spite of these reservations, a prediction based on performance in such apparatus is generally superior to that seen in compendial apparatus based on acids and buffers.

Parts or all of an oral formulation must dissolve in the gastrointestinal contents and the active pharmaceutical ingredient pass from lumen to the absorbing surface. Drugs are not absorbed to any great extent in the stomach and therefore the rate of entry into the duodenum controls the onset of action of immediate release, well-absorbed drugs. As a consequence, there is little need for extensive in vitro examination and simple measures such as disintegration time may be sufficient to characterise the API release from the dosage form in a conventional dissolution apparatus. In contrast, sustained-release formulations may encounter situations which cause altered release; for example, those formulations which are sensitive to alcohol. Here, the physical presentation of the dose form may be responsible for important differences in robustness. As an example, physical compression of the dosage form occurs in the distal stomach, which may be sufficient to cause structural weaknesses in an intact formulation exposed to ethanol - containing media.

In most cases, the interest centres on the processes during the transfer of the formulation from stomach to intestine. Changes in solubility stimulated by $\mathrm{pH}$ may cause precipitation; if this is avoided by supersaturation i.e. in the presence of an excipient, then careful examination of the process to maximise passive Fickian drive maybe justified. Such a process does lend itself to advanced physicochemical investigation to dissect specific API - excipient interactions.

\section{Small Intestine}

In the intestine, the glandular secretory epithelium of the stomach abruptly alters to an absorptive epithelial lining with deep folds and villi. Neutralisation of gastric acid occurs in the shortest section of the intestine and transit through the duodenal region is quick typically less than five minutes. In fasted conditions, formulations are moved through the intestinal by waves of contraction originating from the fundal region, propagated through to the terminal ileum. In clinical trials where the volunteer remains fasted, stasis of the formulation can often be seen in the ileum about two to three hours after ingestion: most water has been absorbed and shear forces are probably low. Feeding causes many 
physiological changes including increased splanchnic blood flow to the upper Gl regions, fat intake release of bile and changes in the intestinal motility to generate more segmenting movements. Dietary fat is an essential substrate in a variety of functions including energy supply and vitamin absorption. During this time there will be movement of water across the gut wall as food is broken down to small more osmotically active fragments causing secretion and small nutrient absorption occurs with water drag of the solutes.

For dissolution and disintegration processes, the aspects regarding absorption, transporters and efflux are not important although there have been attempts to combine models which utilise all the information. Generally, suitable cell lines are used to characterise apical and basal transfer of API from and into the lumen. In silico models are then constructed which utilise all of the information available in order to make predictive simulations. Improvement of biorelevant dissolution fluids and apparatus, which sequentially exposes the drug to differences in luminal composition, suffice to examine rates of dissolution of a particular drug. Thus, for conventional drugs which follow solute-dominated pathways and partition across membranes, the perspective for optimized in vitro approaches is high. This is the objective of the Oral biopharmaceutics tools Initiative, a collaborative venture between industry and academia to develop new instrumentation and computer models, attempting to fill knowledge gaps relating to oral drug delivery (Lennernäs et al, 2014). Recent research in the OrBiTo program has attempted to more closely define the composition of fluids in the distal gut in order to be able to improve biorelevance.

\section{Solubility, Dissolution, Surfactants and Gut contents}

A drug's solid state properties such as particle size or crystalline form (as a polymorph or hydrate) influence the initial dissolution rate and can lead to the generation of supersaturated solutions with solubility greater than the equilibrium value (Shefter \& Higuchi, 1963). The control and modification of both particle size and crystalline form using processes such as comminution therefore can be employed to influence dissolution of poorly soluble drugs such as digoxin and steroids (Florence \& Salole, 1976). The provision of bile salts as natural surfactants to assist in the digestion of fats on drug dissolution was recognized by Borgstrom. He proposed a possible role of bile 
as the medium responsible for increasing the solubility of drugs and other water insoluble substances in gastrointestinal fluid by generation of micellar solutions (Borgstrom, 1962). The micellar assembly of surfactants, the thermodynamic driving forces for this and the ability of these systems to solubilise drugs and impact on formulation problems has been a constant theme of Professor Florence's research (Elworthy \& Florence 1963, Elworthy, Florence \& Macfarlane 1968, Attwood \& Florence 1983). Even in these early publications, it was recognized that, "the use of solubilizers in pharmacy to increase the solubility of sparingly soluble drugs has wide implications, and it is important that these are understood", a task that continues today ( Khadra et al., 2014, Fuchs et al., 2015).

\section{Endogenous Surfactants in the Gut}

The impact of bile salts on drug solubility in the gut was an early area of biopharmaceutical research with studies indicating that dissolution of poorly soluble drugs was enhanced in the presence of bile salts (Bates et al., 1966a), a result of such importance that it warranted publication in a high impact journal! Studies also indicated that solubilisation capacity was bile salt concentration dependent and influenced by the bile salt employed. Additionally, digestion products for example phospholipids and fatty acids impact on gut solubility and therefore the bioavailability of poorly soluble drugs (Charman et al., 1993).

Interest in the role of the gut microbiome in health and disease has revealed significant signaling effects of bile acids on the composition of the colon microbiota, in addition to direct effects on the membrane G-protein coupled receptors which regulate several host pathways (Wahlström et al., 2016). Short term changes in diet, e.g. to entirely animal or vegetarian sources overwhelms the balance of the microbiota and bacteria, viruses and fungi from the new dietary source transiently colonises the colon (David et al., 2014). This indicates a dynamic balance between surfactant presentation and the diversity of our microbial lodgers. If we add to this scenario the exposure to mixes of natural and synthetic surfactants, some of which will travel unaltered to the colon, it will be appreciated that opportunities for surfactant-microbiome interactions are yet to be discovered (see Figure 1). 
Figure 1 near here.

\section{Surfactants in Dissolution}

In 1897 Noyes and Whitney published the first analysis of the dissolution of sparingly soluble compounds and introduced a mathematical analysis demonstrated that the rate of solution of a solid in a bulk dissolution medium was dependent upon the compound's saturation solubility (Noyes \& Whitney,1897). In the 1950's and 1960's, the biopharmaceutical implication of this relationship with respect to the oral administration of drugs was recognized along with examples of differences in bioavailability due to formulation or batch-to-batch variations in performance. In the 1970 's the first pharmacopoeial dissolution tests were introduced in recognition of the importance of this property and in an attempt to apply quality control procedures to oral dosage form performance. In 1995 Amidon introduced the Biopharmaceutical Classification System categorising drugs based on their aqueous solubility and intestinal permeability. Compounds with a low aqueous solubility present the greatest formulation and biopharmaceutical challenge in vivo, and in vitro during dissolution testing. For low solubility compounds, the in vitro dissolution required the development of media with increased solubility either through the use of solutions of single synthetic surfactants or simulated intestinal media (Dressman et al., 1998). The former is simple and illustrate that increased solubility through non-ionic surfactants such as polysorbate 80 increases dissolution, although the surfactant will also affect surface wetting (Mysels \& Florence 1970). Biorelevant media which mimic the natural surfactants and conditions present in the gut are complex mixtures incorporating bile salt, lecithin, fatty acid and monoglyceride and it is possibly not surprising that multiple recipes and interations have been examined during attempts to develop correlations between in vivo performance and in vitro dissolution (Fuchs et al., 2015). Early studies also indicated that this was a complex phenomenon with mixtures of endogenous surfactants exhibiting solubility variations that were drug and mixture specific (Bates et al., 1966b). There is a complex interplay between the drug and surfactant system (Reymond \& Sucker, 1988) and research into this relationship continues. 
In-vitro characterisation of lipid-based formulations (LBFs) presents significant challenges and a simple phosphate buffer dissolution experiment is often not representative of the complex environment of the small intestine. In response to lipid ingestion, secretions of pancreatin and bile salts from pancreas and gall bladder respectively work to digest the lipids, creating a digestion product composed of mixed micelles and bile salt complexes. These mixed micelles act to preserve the drug molecule in the solubilised state and can carry the drug to the site of absorption in the small intestine. For this reason, the representation of the gut in the beaker, particularly the small intestine, requires a more complex media and enzymatic reactions are required to be taken into account particularly for lipid-based formulations.

Representative in-vivo characterisation is by no means novel and historically has been investigated by a number of groups, with a common goal of achieving a global in-vitro model, which is predictive of in-vivo digestion and absorption. Typically, LBFs are assessed in-vitro through dispersion and digestion tests, to investigate emulsification behaviour and solution stability. Initially, dispersion testing is performed in order to understand the stability of LBFs on dilution and likelihood of the API to precipitate from the emulsion. Digestion testing is then used in an attempt to understand the behaviour of the API within the formulation during digestion in the intestine. Early investigational work concerning digestion dates back to the 1980's, where data from in-vitro lipolysis of medium chain triglycerides (MCT) and long chain triglycerides (LCT) were reported (Reymond \& Sucker, 1988). Further models were then developed which described the solubilisation of poorly water-soluble substances during digestion in lipid-based formulations (Christensen et al 2004, Porter et al 2004, Cuine et al., 2008, Sassene et al 2010, Larsen et al.,2011). More recently, the desire within the scientific community to develop standardised in-vitro methods for characterisation and assessment of LBFs, led to the formation of the Lipid Formulation Classification System Consortium (LFCS Consortium). The LFCS Consortium has combined academics and industrial researchers to create a collaborative programme, which is focused on the design of LBF characterisation methods that are both physiologically relevant and applicable to LBF during the developmental phases, potentially leading through to commercialisation. Initial work focused on the establishment of baseline conditions and media selection through the assessment of various LBFs, including type I, II, II and IV LBFs (Williams et al., 2012a) as illustrated in Table 1. 
Table 1 near here...

The concentration of bile salt and drug loading has been investigated with respect to the generation and sustainment of supersaturation of a model poorly soluble API, danazol (Williams et al., 2012b). Bile salt concentration was found to influence the in-vitro digestion of type I and II LBFs; however, the impact of concentration on type III LBF's was not marked due to speed of digestion. In addition, danazol solubilisation within the digested aqueous phase increased with increasing bile salt concentration, up to a concentration of $3 \mathrm{mM}$. The potential for danazol to be maintained as a supersaturated solution within digested formulations, without precipitation, was also characterised. These initial findings were expanded incorporating alternative model APIs fenofibrate and tolfenamic acid (Williams et al., 2013), to help confirm the proposal that the maximum drug supersaturation ratio $\left(\mathrm{SR}^{\mathrm{M}}\right)$ can help define the limitations of supersaturation in in-vitro digested LBFs. The $\mathrm{SR}^{\mathrm{M}}$ is determined from the ratio of maximum theoretical API concentration in the aqueous phase following LBF digestion to maximum API solubility within the digested aqueous phase. It has been suggested by the LFCS Consortium that in order to maintain supersaturation and to limit precipitation, when defining maximum API loading within LBFs the calculated $\mathrm{SR}^{\mathrm{M}}$ should not exceed 3 . In the final part of the series, more challenging conditions for the digestion test were proposed in an attempt to further understand the impact on maintenance of supersaturation and provide discriminatory tests between formulations behaving similarly with standard digestion testing (Williams et al., 2014, BakalaN'Goma et al., 2015). Following this extensive development, the methods refined by the LFCS Consortium have been used routinely in both academia and industry. Although these methods have been of great benefit, challenges remain in the characterisation of some LBFs, including the lack of sink conditions as would be expected during the absorption process, the influence of API metabolism on in-vivo performance and the prediction of performance of LBF suspension formulations. Introducing sink conditions during assessment of LBFs, whilst representing the gut in a beaker is particularly challenging. 


\section{Surfactants incorporated into the Formulation}

The application of surfactants for the solubilisation of materials can be traced back to the 1890's with studies on bile salts and soaps but it was not until the 1930's that these observations were related to the thermodynamically formed colloidal micellar phase. The study of surface tension lowering excipients in pharmaceutical systems started with poorly soluble steroid hormones (Ekwall \& Sjoblom, 1949) but as reviewed by Elworthy, Florence and Mcfarlane in 1968 and again in 1983 (Attwood and Florence) rapidly expanded to cover multiple drugs, dosage routes and solubilisation systems. Initial application in medicines development was limited by the systemic toxicity of the ionic surfactant systems available and it was only on the development of the less toxic non-ionic surfactants that wider pharmaceutical application of solubilized formulations was possible. The physicochemical characterisation of non-ionic surfactants is a research area that was initiated by Sandy and has been a continual focus of his career. The solubilisation and formulation of poorly drugs in surfactant systems was reviewed by Professor Florence in 1981 and this work providing more detail on the influence of the hydrocarbon portion of nonionic surfactants on solubilisation (Florence 1981a). Since that initial review, the number of surfactants available and systems studied has expanded considerably and along with "simple" micellar solubilisation, polymeric micelles and lipid based drug delivery systems (LBDDS) have been developed (Cannon \& Long 2008). Several marketed products incorporate this approach; for example, cyclosporine formulations utilize either corn or olive oil with mixtures of various non-ionic surfactants. Polymeric micelles formed using block co-polymers have mainly been applied to parenteral systems and can be employed for controlled release (Law, Florence \& Whateley 1986). The chemical variability associated with surfactants and the development of chemical coupling systems permits the generation of new supramolecular surfactant-polymer systems. (Al-Jamal, Sakthivel\& Florence, 2005). It is not yet known if these systems will find utility in marketed oral dose products.

\section{Surfactants, enhanced absorption and toxicity}

The ability of non-ionic surfactants which are present in a formulation to enhance the oral absorption of drugs, over and above that associated with solubilisation, has been 
previously reported in the literature (van Hoogdalem, de Boer \& Breimer 1989).

This property is most obvious with drugs which would normally have low or negligible bioavailability when administered orally, for example the oral absorption of methotrexate can be increased by co-administration with polysorbate 80 (Azmin et al 1982). There is however no molecular specificity in this effect and the absorption of poisons is equally promoted to that of the drug (Walters, Dugard, \& Florence 1981). The magnitude of this effect is surfactant concentration-dependent and is linked to the presence of surfactant monomers in solution, leading to activity below the critical micelle concentration $(\mathrm{CMC})$. Above the $\mathrm{CMC}$, solubilisation into the micelle reduces the effect (Florence 1981b) whilst the free monomer concentration is constant. The effect is due to an interaction of the free surfactant monomers with the gut wall epithelial lining. As discussed previously, the membrane is a complex structure consisting of multiple lipid components and proteins. Penetration of the hydrophobic portion into the outer surface of the bilayer disturbs function, increasing absorption. This effect can be applied in multiple drug delivery problems to enhance absorption or tissue penetration (Kerr et al., 1987). However, the negative effect of this interaction is the potential toxicity to the normal physiological function of the membrane (Baillie et al., 1989), which will have to be balanced against increased bioavailability. Additionally, since the surfactants are not selective, the "reduction" of barrier function will not be specific and may also increase the availability of potentially toxic agents from the gut (Al-Jamal, Sakthivel \& Florence, 2005).

\section{Surfactants: the future}

Since the early studies on the influence of surfactants, including natural or synthetic substances, the knowledge base concerning the absorption of poorly soluble drugs from the gut has expanded considerably. Professor Florence's multiple and varied contributions to this field have provided important foundations.

The role of endogenous surfactants such as bile salts, phospholipids and fatty acids (either free or esterified) has been rationalized and simulated dissolution media encompassing fasted and fed states are available. These can be employed to examine the dissolution of poorly soluble drugs and formulations to provide a degree of in vivo prediction. However, the limitations of these systems is apparent (Bergstrom et al., 
2014) and in vivo studies expose the boundaries of current knowledge with respect to the composition of gut fluids (Riethorst et al., 2016) and reveal the impact this might have on solubilisation and absorption.

The availability and use of the non-ionic surfactants, as formulation excipients for drug delivery by all routes has expanded massively in the half-century since Professor Florence published his first manuscript in the field. The use of surfactants in oral formulations either alone, in combination or with lipid-based excipients is now established with multiple marketed formulations for poorly soluble drugs based on these systems. Current research is focused on expanding this armamentarium with more novel combinations of hydrophilic entities with the almost standard hydrophobic long chain fatty acids.

Whilst the experimental knowledge base has expanded, the theoretical models available to determine the nature and extent of the interactions between a surfactant and a poorly soluble molecule in a largely aqueous system remain rooted in basic thermodynamics. A comprehensive treatment of the situation is complicated by the chemical variability of the surfactants (the nature of the hydrophobic and hydrophilic groups), the inherent concentration and component variability of the gut. These are complex systems and it has long been recognized that phase changes and anisotropic behavior are normal which increase the difficulty in prediction of performance. The challenge for the next half century must be to develop a suitable predictive theoretical framework, a situation presaged by Professor Florence in 1968 when he wrote with respect to surfactants that "a great deal of work using modern methods is still required to clarify the situation" (Florence, 1968). 


\section{References}

Al-Dujaili, H., Florence, A. T., Salole, E. G., 1986. The adhesiveness of proprietary tablets and capsules to porcine oesophageal tissue. Int. J. Pharm. 34, 75-79.

Al-Jamal, K. T., Sakthivel, T., Florence, A. T., 2005. Solubilisation and transformation of amphipathic lipidic dendron vesicles (dendrisomes) into mixed micelles. Colloids and Surfaces A: Physicochemical and Engineering Aspects, 268, 52-59.

Attwood, D.,Florence,A.T., 1983. Surfactant Systems: Their Chemistry, Pharmacy and Biology, Chapman and Hall Ltd, London, 1983.

Azmin, M. N., Stuart, J. F., Calman, K. C. et al.,1982. Effects of polysorbate 80 on the absorption and distribution of oral methotrexate (MTX) in mice. Cancer chemotherapy and pharmacology, 9, 161-164.

Baillie, A. J., Al-Assadi, H., Florence, A. T.,1989. Influence of non-ionic surfactant structure on motility inhibition of Tetrahymena elliotti: a model for surfactant-membrane interactions. Int. J. Pharm. 53, 241-248.

Bakala-N'Goma, J. C., Williams, H. D., Sassene, P. J., et al., 2015. Toward the establishment of standardized in vitro tests for lipid-based formulations. 5. Lipolysis of representative formulations by gastric lipase. Pharm. Res.32, 1279-1287.

Bates, T. R., Gibaldi, M., Kanig, J. L., 1966a. Solubilizing properties of bile salt solutions I: Effect of temperature and bile salt concentration on solubilization of glutethimide, griseofulvin, and hexestrol. J. Pharm. Sci. , 55, 191-199.

Bates, T. R., Gibaldi, M., \& Kanig, J. L., 1966b. Solubilizing properties of bile salt solutions I: Effect of temperature and bile salt concentration on solubilization of glutethimide, griseofulvin, and hexestrol. J. Pharm. Sci. 55, 191-199 
Bergström, C. A., Holm, R., Jørgensen, S. A.,et al., 2014. Early pharmaceutical profiling to predict oral drug absorption: current status and unmet needs. Eur. J. Pharm. Sci., 57, 173199.

Borgstrom, B. (1962). Digestion and absorption of fat. Gastroenterology, 43, 216-219.

Butler, J.M., Dressman, J.B. 2010. The Developability Classification System: Application of Biopharmaceutics Concepts to Formulation Development. J. Pharm. Sci., 99, 4940-4954

Cannon, J.B., Long, M.A. 2008. Emulsions, Microemulsions, and Lipid-Nased Drug Delivery Systems for Drug Solubilization and Delivery - Part II Oral Applications, in: R. Liu (Ed.) Water Insoluble Drug Formulation, CRC Press, Boca Raton, 2008, pp. 227-254.

Charman, W. N., Rogge, M. C., Boddy, A. W.,et al.,1993. Effect of food and a monoglyceride emulsion formulation on danazol bioavailability. J.Clin. Pharmacol. 33, 381386.

Christensen, J. Ø., Schultz, K., Mollgaard, B., et al., 2004. Solubilisation of poorly watersoluble drugs during in vitro lipolysis of medium-and long-chain triacylglycerols. Eur. J. Pharm. Sci. 23, 287-296.

Clavé, P., Arreola, V., Romea, M.,et al., 2008. Accuracy of the volume-viscosity swallow test for clinical screening of oropharyngeal dysphagia and aspiration. Clin. Nutr. 27, 806-815.

Cuine, J. F., McEvoy, C. L., Charman, W. N.et al., 2008. Evaluation of the impact of surfactant digestion on the bioavailability of danazol after oral administration of lipidic selfemulsifying formulations to dogs. J. Pharm. Sci. 97(2), 995-1012.

David, L. A., Maurice, C. F., Carmody, R. N. et al.,2014 . Diet rapidly and reproducibly alters the human gut microbiome. Nature, 505, 559-563. 
Dressman, J. B., Amidon, G. L., Reppas, C., et al.,1998. Dissolution testing as a prognostic tool for oral drug absorption: immediate release dosage forms. Pharm. Res. 15, 11-22.

Dressman, J.B., Vertzoni, M., Goumas,K., et al., 2007. Estimating drug solubility in the gastrointestinal tract, Adv. Drug Deliv. Rev. 59,591-602.

Duggan, J. M., Shukla, V., Akpanudo, B., et al., 2014. Doc, I Just Can't Swallow Pills": HIV Infected Patients and Pill Phagophobia. J. AIDS Clin Res. 5:348 doi:10.4172/21556113.1000348

Efentakis, M., Dressman, J.B., 1988. Gastric juice as a dissolution medium: surface tension and $\mathrm{pH}$. Eur. J. Drug Met. Pharmacokin. 23, 97-102.

Ekwall, P., Sjoblom, L., 1949. On the solubilization of steroid hormones by association colloids. Acta Chemica Scand. 3, 1179-1180.

Elworthy, P.H., Florence,A.T., 1963. Thermodynamics of micelle formation of non-ionic detergents, J.Pharm. Pharmacol. 15, 851-852.

Elworthy, P.H., Florence, A.T., Macfarlane, C.B. 1968 Solubilization by surface active agents and its applications in chemistry and the biological sciences., Chapman and Hall, London, 1968.

Fell, J. T., Mohammad, H.A.H., 1995. The wetting of powders by bile salt solutions and gastric juice. Int. J. Pharm. 125, 327-330.

Finholt, P.,Solvang, S., 1968. Dissolution kinetics of drugs in human gastric juice-the role of surface tension. J. Pharm. Sci. 57, 1322-1326.

Florence, A.T., Salole, E. G., 1976. Changes in crystallinity and solubility on comminution of digoxin and observations on spironolactone and oestradiol. J. Pharm. Pharmacol. 28, 637642. 
Florence, A.T.,1968. Surface chemical and micellar properties of drugs in solution. Adv. Colloid Interface Sci., 2, 117-149.

Florence, A.T.,1981a. Drug solubilisation in surfactant systems., in: S. Yalkowsky (Ed.) Techniques of solubilisation of drugs, Marcel Dekker, New York, 1981.

Florence, A. T.,1981b. Surfactant interactions with biomembranes and drug absorption. Pure and Applied Chemistry, 53(11), 2057-2068.

Fuchs, A., Leigh, M., Kloefer, B., et al., 2015. Advances in the design of fasted state simulating intestinal fluids: FaSSIF-V3. Eur. J. Pharm. Biopharm. 94, 229-240.

Garbacz G., Wedermeyer R.S., Stefan N et al., 2008. Irregular absorption profiles observed from diclofenac extended release tablets can be predicted using a dissolution apparatus that mimics in vivo stresses. Eur. J. Pharm. Biopharm. 70: 421-428.

Gleeson, D. C., 1999. Oropharyngeal swallowing and aging: a review. J. Communication Disorders, 32,373-396.

Kerr, D. J., Wheldon, T. E., Russell, J. G., et al., 1987. The effect of the non-ionic surfactant brij 30 on the cytotoxicity of adriamycin in monolayer, spheroid and clonogenic culture systems.Eur. J. Cancer. Clin. Oncol. 23, 1315-1322.

Khadra I., Zhou Z., Dunn C.,et al., 2014. Statistical investigation of simulated intestinal fluid composition on the equilibrium solubility of biopharmaceutics classification system class II drugs . Eur. J. Pharm. Sci., 67, 65-75.

Larsen, A. T., Sassene, P., Müllertz, A. 201). In vitro lipolysis models as a tool for the characterization of oral lipid and surfactant based drug delivery systems. Int. J. Pharm., 417, 245-255. 
Law, T. K., Florence, A. T., Whateley, T. L., 1986. Some chemically modified poloxamer hydrogels: controlled release of prostaglandin-E 2 and testosterone. Int. J. Pharm., 33, 65-69.

Lennernäs H., Aarons, L., Augustijns, P. et al., 2014. Oral biopharmaceutics tools- Time for a new initiative - an introduction to the IMIM project. Eur. J. Pharm. Sci., 57, 292-299.

Marques, M.R.C., Loebenberg,R. , Almukainzi, M. 2011. Simulated biological fluids with possible application in dissolution testing. Dissolution Technol 18, 15-28.

Mojet, J., Christ-Hazelhof, E., Heidema, J., 2001. Taste perception with age: generic or specific losses in threshold sensitivity to the five basic tastes?. Chemical Senses, 26, 845-860.

Mysels, K. J., Florence, A. T. 1970. Clean Surfaces: Their Preparation and Characterization for Interfacial Studies in 'Techniques and criteria in the purification of aqueous surfaces' Goldfinger E. (Ed) Marcel Dekker, New York, pp 227-268.

Nelson, E.,1957. Solution rate of theophylline salts and effects from oral administration. J. Pharm. Sci. 46, 607-614.

Noyes, A.N., Whitney W.R., 1897. The rate of solution of solid substances in their own solutions. J. Am. Chem. Soc 19, 930-934.

O'Reilly S., Wilson C.G., Hardy J.G., 1987. The influence of food on gastric emptying of multiparticulate dosage forms. Int. J. Pharm. 34, 213-216

Perkins, A. C., Wilson, C. G., Blackshaw, P. E., et al., 1994. Impaired oesophageal transit of capsule versus tablet formulations in the elderly. Gut, 35, 1363-1367.

Perkins, A. C., Wilson, C. G., Frier, M., 2001. The use of scintigraphy to demonstrate the rapid esophageal transit of the oval film-coated placebo risedronate tablet compared to a round uncoated placebo tablet when administered with minimal volumes of water. Int. J. Pharm. 222, 295-303. 
Pohle, T., Domschke, W., 2003. Gastric function measurements in drug development. Br. J. Clin. Pharmacol. 56, 156-164

Porter, C. J., Kaukonen, A. M., Taillardat-Bertschinger, A., et al.,2004. Use of in vitro lipid digestion data to explain the in vivo performance of triglyceride- based oral lipid formulations of poorly water- soluble drugs: studies with halofantrine. J. Pharm. Sci. 93, $1110-1121$.

Reymond J.P., Sucker H., 1988. In vitro model for ciclosporin intestinal absorption in lipid vehicle. Pharm. Res. 5, 673-6.

Riethorst, D., Mols, R., Duchateau, G. et al., 2016. Characterization of human duodenal fluids in fasted and fed state conditions. J. Pharm. Sci. 105, 673-681.

Sassene, P. J., Knopp, M. M., Hesselkilde, J. Z., et al., 2010. Precipitation of a poorly soluble model drug during in vitro lipolysis: characterization and dissolution of the precipitate. J. Pharm. Sci. 99, 4982-4991

Shefter, E., Higuchi, T., 1963. Dissolution behavior of crystalline solvated and nonsolvated forms of some pharmaceuticals. J. Pharm. Sci. 52, 781-791.

Stegemann, S., Ecker, F., Maio, M. et al., 2010. Geriatric drug therapy: Neglecting the inevitable majority. Ageing Res. Rev., 9, 384-398.

Swisher, D. A., Sendelbeck, S. L., Fara, J.W., 1984. Adherence of various oral dosage forms to the esophagus. Int. J. Pharm. 22, 219-228.

van Hoogdalem, E. J., de Boer, A. G., Breimer, D. D., 1989. Intestinal drug absorption enhancement: an overview. Pharmacol. Ther. 44, 407-443.

Vingerhoeds, M. H., Blijdenstein, T. B., Zoet, F. D.,et al., 2005. Emulsion flocculation induced by saliva and mucin. Food Hydrocolloids, 19, 915-922. 
Walters, K. A., Dugard, P. H., Florence, A. T.,1981. Non- ionic surfactants and gastric mucosal transport of paraquat1. J. Pharm. Pharmacol. 33, 207-213.

Wahlström, A., Sayin, S. I., Marschall, H. U.,. et al., 2016. Intestinal crosstalk between bile acids and microbiota and its impact on host metabolism. Cell Metabolism, 24, 41-50.

Washington, N., Wilson, C.G., Davis, S.S., 1985. Evaluation of 'raft-forming'antacid neutralizing capacity: in vitro and in vivo correlations. Int. J. Pharm. 27, 279-286.

Williams, H. D., Sassene, P., Kleberg, K. et al., 2012a. Toward the establishment of standardized in vitro tests for lipid-based formulations, part 1: method parameterization and comparison of in vitro digestion profiles across a range of representative formulations. J. pharm. Sci. 101, 3360-3380.

Williams, H. D., Anby, M. U., Sassene. et al., 2012b. Toward the establishment of standardized in vitro tests for lipid-based formulations. 2. The effect of bile salt concentration and drug loading on the performance of type I, II, IIIA, IIIB, and IV formulations during in vitro digestion. Mol. Pharmaceut. 9, 3286-3300.

Williams, H. D., Sassene, P., Kleberg, K. et al., 2013. Toward the establishment of standardized in vitro tests for lipid-based formulations, part 3: understanding supersaturation versus precipitation potential during the in vitro digestion of type I, II, IIIA, IIIB and IV lipidbased formulations. Pharm. Res. 30, 3059-3076.

Williams, H. D., Sassene, P., Kleberg, K., et al., 2014. Toward the Establishment of Standardized In Vitro Tests for Lipid- Based Formulations, Part 4: Proposing a New Lipid Formulation Performance Classification System. Journal of pharmaceutical sciences, 103(8), 2441-2455. 
Illustrations

\begin{tabular}{|c|c|c|c|c|c|}
\hline \multirow{2}{*}{$\begin{array}{l}\text { EXCIPIENTS IN } \\
\text { FORMULATION }\end{array}$} & \multicolumn{4}{|c|}{ CONTENT OF FORMULATION ( $\%$, W/W ) } & \multirow[b]{2}{*}{$\begin{array}{c}\text { TYPE IV } \\
\text { OIL-FREE }\end{array}$} \\
\hline & $\begin{array}{l}\text { TYPE } 1 \\
\text { OIL }\end{array}$ & $\begin{array}{l}\text { TYPE II } \\
\text { SEDDS }\end{array}$ & $\begin{array}{l}\text { TYPE IIIA } \\
\text { SEDDS }\end{array}$ & $\begin{array}{l}\text { TYPE IIIB } \\
\text { SMEDDS }\end{array}$ & \\
\hline $\begin{array}{l}\text { Oils:tri,di \& } \\
\text { monoglycerides }\end{array}$ & 100 & $40-80$ & $40+80$ & $<20$ & - \\
\hline $\begin{array}{l}\text { Water-insoluble } \\
\text { surfactants }\end{array}$ & - & $20-60$ & - & - & $0-20$ \\
\hline $\begin{array}{l}\text { Water-soluble } \\
\text { surfactants }\end{array}$ & - & - & $20-40$ & $20-50$ & $30-80$ \\
\hline $\begin{array}{l}\text { Hydrophilic } \\
\text { cosolvents }\end{array}$ & - & - & $0-40$ & $20-50$ & $0-50$ \\
\hline $\begin{array}{l}\text { Types of } \\
\text { dispersion }\end{array}$ & $\begin{array}{l}\text { Limited or no } \\
\text { dispersion }\end{array}$ & $\begin{array}{c}\text { Rapidly } \\
\text { dispersing }\end{array}$ & $\begin{array}{l}\text { Rapidly } \\
\text { dispersing }\end{array}$ & $\begin{array}{l}\text { Transparent } \\
\text { dispersion }\end{array}$ & $\begin{array}{l}\text { Micellar } \\
\text { solution }\end{array}$ \\
\hline $\begin{array}{l}\text { Digestion } \\
\text { requirement }\end{array}$ & $\begin{array}{l}\text { Requires } \\
\text { digestion }\end{array}$ & $\begin{array}{l}\text { Likely to be } \\
\text { digested }\end{array}$ & $\begin{array}{c}\text { Dige } \\
\text { not be }\end{array}$ & $\begin{array}{l}\text { tion may } \\
\text { eecessary }\end{array}$ & $\begin{array}{c}\text { Limited } \\
\text { digestion }\end{array}$ \\
\hline
\end{tabular}

Table 1. Types of LBF as proposed by the LBF Consortium (adapted from Williams 2012a) 


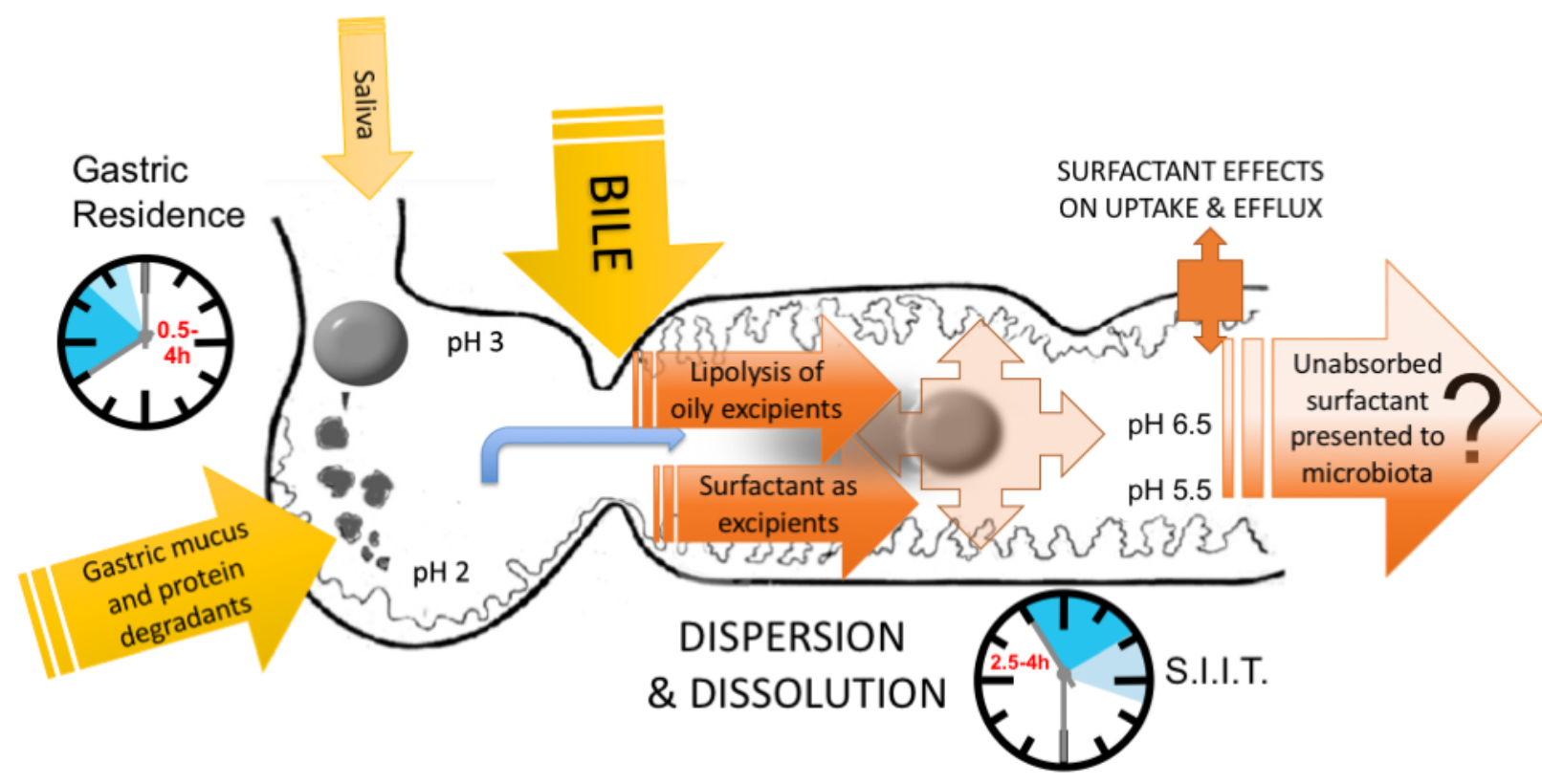

Figure $1 \&$ Graphical abstract. Presentation of surfactants to the gut shows dynamic alterations in composition, reflecting digestion of fats, release of excipients and absorption along the gut 